\title{
Monitoring a gas-cooled nuclear reactor core integrity.
}

\author{
Claudio Bonivento* Michael J. Grimble** \\ Leonardo Giovanini ${ }^{* *}$ Mattia Monari ${ }^{* * *}$ Andrea Paoli ${ }^{*, 2}$ \\ * Center for Research on Complex Automated Systems (CASY), DEIS, \\ University of Bologna, Viale Pepoli, 3/2 - 40136 Bologna, ITALY, \\ \{claudio.bonivento; andrea.paoli\}@unibo.it \\ ** Industrial Control Center (ICC), Strathclyde University of Glasgow, \\ Graham Hills Building, 50 George Street, Glasgow G1 1QE, Scotland, \\ $U K,\{$ m.grimble ; leonardo.giovanini\}@eee.strath.ac.uk \\ *** Former visiting student at ICC, mattia.monari@aliceposta.it
}

\begin{abstract}
This work concerns with the problem of monitoring an Advanced Gas-cooled Nuclear Reactor (AGR) core. In the world wide context of energy production, the advanced gas-cooled reactor (AGR) nuclear power stations are approaching the end of their predicted operational live. Currently, it has been proposed to extend the operational lifetime of the nuclear plants if the distortions of the reactor cores are not as severe as initially predicted, and if it is possible to prove that the reactors are still safe to operate. The purpose of this work is to present a monitoring system based on analytical redundancy and directional residual generation using measurements obtained during the refueling process. In short this problem consists of building an unknown input observer with the role to estimate the friction force produced by the interaction between the wall of the fuel channel and the fuel assembly. This let to estimate the shape of the graphite bricks that comprise the core monitoring any distortion of them. Copyright (C)2008 IFAC.
\end{abstract}

Keywords: Security monitoring, Nuclear reactors, Kalman filtering, Quantization.

\section{INTRODUCTION}

This work concerns with the problem of monitoring an Advanced Gas-cooled Nuclear Reactor (AGR) core. This plant makes use of the heat given by the nuclear efficient reaction to produce electricity by means of steam turbines. These are driven by steam, which is heated, from the AGR gas using a heat exchanger. One of the advantages of a gas cooled reactor is the high temperature that the gas can achieve so that when it is used in conjunction with the heat exchanger and steamed turbine the thermal efficiency is very high.

In the world, and specifically in the United Kingdom, the advanced gas-cooled reactor (AGR) nuclear power stations are approaching the end of their predicted operational live. The reactor core is composed of a hundreds of hollow graphite bricks (that acts as neutron moderator), and the graphite ages because of neutron irradiation and radiolytic oxidation causing distortion and potentially cracking of the bricks. Since it is impossible to repair or replace the graphite bricks the graphite core is one of the main components that determinate the operational life of a nuclear station. In other terms the major factor that dictates the life of a nuclear power station is the condition of the graphite reactor core, which distorts over time with

\footnotetext{
1 Authors wish to thank British Energy Agency and in particular Dr. Jim Reed for making possible a fruitful collaboration and for the permission of using real-plant data.

2 Corresponding author.
}

prolonged exposure to heat and radiation. Currently, it has been proposed to extend the operational lifetime of the nuclear plants if the distortions of the reactor cores are not as severe as initially predicted, and if it is possible to prove that the reactors are still safe to operate. From this, it is clear how important is to keep under monitoring the integrity of the plant and especially of the core; this is actually made possible by a routine performed during planned station outage. These outages occur roughly every three years and result in a large volume of detailed information collected by a system called Channel Bore Monitoring Unit (CBMU). This data consists of accurate measures of the channel bore diameter and tilt angles; this information is used to provide an overall assessment

To perform a more accurate monitoring of the core over their predicted operational life, the estimation of its state should be more frequent; on the other side it is important that the reactor is not offline frequently or for long periods. On the other hand data is also gathered during core refueling operations, i.e. when the uranium dioxide fuel is replaced with a weekly rate. An important source of information during the refueling phase is the fuel grab load trace data, that consists in collecting information on the position of the uranium bar inserted in the core and information on the force produced by the interaction between the wall of the fuel channel and the fuel assembly supporting brushes. Since interfaces between adjacent brick layers result in changes in the bore diameter of the channel, as the brushes supporting the fuel rods pass through these 
features, there is an equivalent change in the friction forces between the walls and the brushes, which correspond to an apparent change of the load force on the fuel assembly. This change in load manifests itself as peaks within the refueling load trace. In West et al. [2005b], CBMU data was compared with load trace data coming from different refueling event and it has been shown how a load trace and a CMBU trace can furnish the same information (see Pang et al. [2006], Pang et al. [2007] and West et al. [2005a]).

Following these considerations, the purpose of this work is to explore a monitoring system based on analytical redundancy and directional residual generation using measurements obtained during the refueling process. In short this problem consists of building an unknown input observer with the role to estimate the friction force produced by the interaction between the wall of the fuel channel and the fuel assembly supporting brushes. This let to estimate the shape of the graphite bricks that comprise the core and, therefore, monitor any distortion of them. The theoretical machinery exploited is the Kalman filter theory (e.g. Jazwinski [1970],Grawal and Andrews [1993]), which is used to estimate the information above mentioned. In a different nuclear context, in particular in safeguards problems, a similar approach has been used in Bonivento [1983]. In this paper we will discuss the model of the system used for estimation purposes and the application of a discrete-time Kalman filter to estimate the friction force from the fuel grab load signal stored during the refueling process. Since the initial condition of the system are not known, and considering the fact that the estimation process is performed off-line, a smoothing algorithm based on Kalman filter is introduced to improve the estimate. This is important as a matter of fact that, even if the grab load data is a time signal, it should be considered as parametrized in the height dimension of the fueling channel wall. Hence a perfect estimate both for $t=0$ and $t=N$ is necessary. Moreover it will be presented how to deal with the quantization of the filtered data that introduce a noise in data streams (see e.g. Ziskand and Hertz [1993]). Some ideas about this approach can be found also in Bonivento et al. [2007].

\section{USING KALMAN FILTER AND SMOOTHER TO ESTIMATE THE CORE CONDITION}

Each refueling phase provides two data traces, one obtained by lowering the fueling assembly into the nuclear core, and the other one by raising the fuel assembly out from there. The fuel grab load trace data is obtained during the refueling by load cells positioned on the refueling machine which directly measure the force applied by the fuel assembly. This force depends on several factors, among which the most significant are in the following described

a) The weight of the fuel assembly: this term depends on the fuel rod mass which changes due to the nuclear reactions in the core.

b) The frictional forces: is the quantity that we want to estimate, is caused by the interaction with the stabilizing brushes on the fuel channel wall: any distortion in the channel geometry will reflects in friction force changes.

c) The buoyancy force: is caused by the gas that, circulating in the fuel chamber, makes the fuel assembly appear lighter. This force is unknown and changes its effect on the fuel assembly with the position of the uranium bar into the channel.

During refueling process, the fuel assembly is governed by the interaction of forces that simultaneously act on the fuel assembly; the simple discrete time model used is:

$$
\begin{aligned}
{\left[\begin{array}{l}
x_{1}(t+1) \\
x_{2}(t+1)
\end{array}\right]=} & {\left[\begin{array}{cc}
1 & 0 \\
\Delta t & 1
\end{array}\right]\left[\begin{array}{l}
x_{1}(t) \\
x_{2}(t)
\end{array}\right]+\Delta t\left[\begin{array}{c}
-1 / m \\
0
\end{array}\right] F_{f}(t)+} \\
& +\Delta t\left[\begin{array}{c}
-F_{l} / m-g \\
0
\end{array}\right]+w(t) \\
y(t)= & {\left[\begin{array}{ll}
1 & 0 \\
0 & 1
\end{array}\right]\left[\begin{array}{l}
x_{1}(t) \\
x_{2}(t)
\end{array}\right]+v(t) . }
\end{aligned}
$$

where $x_{1}$ is the position of the fuel assembly, $x_{2}$ is its speed, $w$ is system noise and $v$ is measurements noise, $m$ is the fuel assembly mass, $g$ is the gravitational acceleration, $F_{l}$ is the grab load force, $F_{f}$ is the brushes friction forces and applied by the supporting brushes on the assembly and $F_{a}$ is the aerodynamic force due to the gas flow in the fuel chamber; moreover $t$ is the discrete time and $\Delta t$ is the sample period.

Aim of this section is to present a 3-step estimation procedure that, starting from model (1) and having available the measure of the position of the fuel assembly along the channel and the grab force applied on it, is able to estimate the friction force $F_{f}$. Considering the friction force $F_{f}$ as an unknown input for system (1) and using an adapted version of Kalman filter for systems with unknown inputs (see Emara-Shabaik [2003]), we will estimate the system state. Having the state estimation it is possible to evaluate the friction force term $F_{f}$ using the first equation in (1). In order to improve the estimation for small time instants (i.e. for the initial position of the fuel assembly), having a first estimation of the unknown input $F_{f}$, it is possible to use a Kalman smoother to process the system in the reverse way, find an estimation of the state and, consequently, of the friction force at time $t=N ; N-1 ; \ldots 1 ; 0$. Finally, in order to find an optimal estimation of the system state, and hence an optimal estimation of the friction force, the system will be processed using a forward known input Kalman filter. Roughly speaking running the Kalman filter forward in time we estimate the state of the system, while running it backward in time we make a correction of the previous estimate of the friction force thanks to additional information of the system gathered during the first forward estimation.

Recalling system (1), our aim is to write it in the form

$$
\begin{aligned}
{\left[\begin{array}{c}
z_{d}(t+1) \\
z_{f}(t+1)
\end{array}\right]=} & {\left[\begin{array}{ll}
F_{1} & F_{2} \\
F_{3} & F_{4}
\end{array}\right]\left[\begin{array}{l}
z_{d}(t) \\
z_{f}(t)
\end{array}\right]+\left[\begin{array}{c}
\bar{D} \\
0
\end{array}\right] d(t)+} \\
& +\left[\begin{array}{l}
\bar{G}_{1} \\
\bar{G}_{2}
\end{array}\right]+w \\
y(t)= & {\left[\begin{array}{ll}
\bar{C}_{1} & \bar{C}_{2}
\end{array}\right]\left[\begin{array}{l}
z_{d}(t) \\
z_{f}(t)
\end{array}\right]+v(t) . }
\end{aligned}
$$

where the disturbance $d(t)$ acts as the friction force $F_{f}$. Defining the non-singular real matrix $U$

$$
U=\left[\begin{array}{cc}
1 & -1 \\
0 & 1
\end{array}\right],
$$

it is possible to find the relation between system (1) and (2): 


$$
\begin{gathered}
{\left[\begin{array}{ll}
F_{1} & F_{2} \\
F_{3} & F_{4}
\end{array}\right]=U\left[\begin{array}{cc}
1 & 0 \\
\Delta t & 1
\end{array}\right] U^{-1}:=\bar{A}} \\
{\left[\begin{array}{c}
\bar{D} \\
0
\end{array}\right]=U\left[\begin{array}{c}
-\Delta t / m \\
0
\end{array}\right]:=\bar{B}} \\
{\left[\begin{array}{ll}
\bar{C}_{1} & \bar{C}_{2}
\end{array}\right]=\left[\begin{array}{ll}
1 & 0 \\
0 & 1
\end{array}\right] U^{-1}:=\bar{C}} \\
{\left[\begin{array}{l}
z_{d}(t) \\
z_{f}(t)
\end{array}\right]=U x(t):=\left[\begin{array}{l}
\bar{x}_{1}(t) \\
\bar{x}_{2}(t)
\end{array}\right] .}
\end{gathered}
$$

Note that the term

$$
\Delta t\left[\begin{array}{c}
-F_{l} / m-g \\
0
\end{array}\right]
$$

in first equation of system (1) is not present in the correspondent equation of system (2); this matrix, referred as $E$, will be consider as a known input of the system (1). Following this reasoning, the system can be rewritten in the form

$$
\begin{aligned}
{\left[\begin{array}{l}
\bar{x}_{1}(t+1) \\
\bar{x}_{2}(t+1)
\end{array}\right] } & =\bar{A}\left[\begin{array}{l}
\bar{x}_{1}(t+1) \\
\bar{x}_{2}(t+1)
\end{array}\right]+\bar{B} F_{f}(t)+\bar{E}+w \\
y(t) & =\bar{C}\left[\begin{array}{l}
\bar{x}_{1}(t+1) \\
\bar{x}_{2}(t+1)
\end{array}\right]+v(t) .
\end{aligned}
$$

where

$$
\bar{E}=U E=\Delta t\left[\begin{array}{c}
-F_{l}-g \\
0
\end{array}\right]
$$

In order to estimate the system state in presence of an unknown input, its effect on the system must be isolated; to this aim it is possible to define a non-singular real matrix $V$, such that

$$
\begin{gathered}
V y(t):=\left[\begin{array}{l}
\bar{y}_{1}(t) \\
\bar{y}_{2}(t)
\end{array}\right]=\bar{y}(t) \quad V \bar{C}=\left[\begin{array}{cc}
\bar{C}_{11} & \bar{C}_{12} \\
0 & \bar{C}_{22}
\end{array}\right] \\
V v(t)=\left[\begin{array}{l}
\bar{v}_{1}(t) \\
\bar{v}_{2}(t)
\end{array}\right]
\end{gathered}
$$

in this way we have transformed the second equation of (9) in the following form:

$$
\begin{aligned}
& \bar{y}_{1}(t)=\bar{C}_{11} \bar{x}_{1}(t)+\bar{C}_{12} \bar{x}_{2}(t)+\bar{v}_{1}(t) \\
& \bar{y}_{2}(t)=\bar{C}_{22} \bar{x}_{2}(t)+\bar{v}_{2}(t) ;
\end{aligned}
$$

where $\bar{C}_{11}$ is a matrix with rank $l$ in order to preserve system observability.

Now it is possible to rewrite the first equation in (12) as

$$
\bar{x}_{1}(t)=\bar{C}_{11}^{-1}\left[\bar{y}_{1}(t)-\bar{C}_{12} \bar{x}_{2}(t)-\bar{v}_{1}(t)\right]
$$

and substituting this into the first equation of (9) it is possible to find that

$$
\begin{aligned}
\bar{x}_{2}(t+1) & =\tilde{A} \bar{x}_{2}(t)+\tilde{B} \bar{y}_{1}(t)+\bar{E}_{2}+\tilde{G} \tilde{w}(t) \\
\bar{y}_{2}(t) & =\bar{C}_{22} \bar{x}_{2}(t)+\bar{v}_{2}(t),
\end{aligned}
$$

where

$$
\begin{aligned}
& \tilde{A}=\left[\bar{A}_{22}-\bar{A}_{21} \bar{C}_{11}^{-1} \bar{C}_{12}\right] \quad \tilde{B}=\bar{A}_{21} \bar{C}_{11}^{-1} \\
& \tilde{G}=\left[\bar{G}_{2}-\bar{A}_{21} \bar{C}_{11}^{-1}\right] \quad \tilde{w}(t)=\left[w(t) \bar{v}_{1}(t)\right]^{\mathrm{T}} .
\end{aligned}
$$

Now it si possible to estimate the state of system (14) applying the known input Kalman filter.

First step (unknown input Kalman Filter)
State estimation a priori:

$$
\hat{x}_{2}(t+1)=\tilde{A} \hat{x}_{2}(t \mid t)+\tilde{B} \hat{y}_{1}(t)
$$

Error covariance a priori:

$$
P_{2}(t+1)=\tilde{A} P_{2}(t \mid t) \tilde{A}^{\mathrm{T}}+Q_{2}
$$

Kalman gain matrix:

$$
K(t+1)=P_{2}(t+1) \bar{C}_{22}^{\mathrm{T}}\left[\bar{C}_{22} P_{2}(t+1) \bar{C}_{22}^{\mathrm{T}}+R_{2}\right]^{-1}
$$

State estimation a posteriori:

$$
\begin{aligned}
\hat{x}_{2}(t+1 \mid t+1)= & \hat{x}_{2}(t+1)+K(t+1)\left[\bar{y}_{2}(t+1)+\right. \\
& \left.-\bar{C}_{22} \hat{x}_{2}(t+1)\right]
\end{aligned}
$$

Error covariance a posteriori:

$$
P_{2}(t+1 \mid t+1)=P_{2}(t+1)-K(t+1) \bar{C}_{2} 2 P_{2}(t+1)
$$

Initial conditions

$$
\hat{x}_{2}(0)=0 \quad P_{2}(0)=1 e 7 .
$$

Having the estimate $\hat{x}_{2}(t)$ it is possible to compute $\hat{x}_{1}(t)$ from equation (13) as

$$
\hat{x}_{1}(t)=\bar{C}_{11}^{-1}\left[\bar{y}_{1}(t)-\bar{C}_{12} \hat{x}_{2}(t \mid t)\right]
$$

with conditional covariance

$$
P_{1}(t)=\bar{C}_{11}^{-1} \bar{C}_{12} P_{2}(t \mid t) \bar{C}_{12}^{\mathrm{T}} \bar{C}_{12}^{\mathrm{T}}{ }^{-1}+\bar{C}_{11}^{-1} R_{1}(t) \bar{C}_{11}^{-1^{\mathrm{T}}},
$$

where $R_{1}(t)$ is the covariance matrix of the noise term $v_{1}(t)$.

From the estimates $\hat{x}_{1}(t)$ and $\hat{x}_{2}(t)$ it is possible to compute the friction force $F_{f}(t)$ using the first equation of (9):

$$
\hat{F}_{f}(t)=\bar{B}^{-1}\left[\hat{x}_{1}(t+1)-\bar{A}_{11} \hat{x}_{1}(t)-\bar{A}_{12} \hat{x}_{1}(t \mid t)-\bar{E}_{1}\right] .
$$

Moreover the estimate state $x(t)$ of system (1) and its error covariant matrix can be computed as:

$$
\hat{x}(t)=U^{-1}\left[\begin{array}{c}
\hat{x}_{1}(t) \\
\hat{x}_{2}(t)
\end{array}\right] \quad P(t)=U^{-1}\left[\begin{array}{cc}
P_{1}(t \mid t) & L(t) \\
L^{\mathrm{T}}(t) & P_{2}(t \mid t)
\end{array}\right]
$$

where $L(t)=-\bar{C}_{11}^{-1} \bar{C}_{12} P_{2}(t \mid t)$. The Kalman filter based algorithm just presented is able to estimate the state of the system even if a disturbance (represented in our case by the friction force determined by the brushes) is acting on it. From this estimate it is possible to compute the magnitude of the friction force $F_{f}$ simply using (9). It is important to note that the statistic property of the friction force at the instant time $t=0$ are not known, and therefore the state estimation in $t=0$ is not appropriate. Remember that this fact reflects in a wrong estimation of the friction force applied on the fuel assembly around its initial position.

The idea to deal with this problem is to use the estimation of the friction force to improve the state estimates just by gathering information in the reverse way. Thus, applying the backward Kalman filter on system (1), here rewritten as

$$
\begin{aligned}
\bar{x}(t+1) & =A \bar{x}(t)+B F_{f}(t)+E(t)+w(t) \\
y(t) & =C \bar{x}(t)+v(t) ;
\end{aligned}
$$

it is possible to obtain the optimal estimate of the friction force at time $t=0$. The backward Markovian model considering now the friction force as a known input is 


$$
\begin{aligned}
\bar{x}_{b}(t) & =A^{-1} \bar{x}_{b}(t+1)-A^{-1} B \hat{F}_{f}(t)-\bar{A}^{-1} E(t)+w(t) \\
y(t) & =C \bar{x}_{b}(t)+v(t)
\end{aligned}
$$

applying the Kalman smoothing algorithm to system (27) it is possible to estimate its state from $t=N$ up to $t=0$ using the following procedure.

\section{Second step (known input Kalman Smoother)}

State estimation a priori:

$$
\hat{x}_{b}(t-1 \mid t)=A^{-1} \hat{x}_{b}(t \mid t)-A^{-1} B F_{f}(t-1)-A^{-1} E(t)
$$

Error covariance a priori:

$$
P_{b}(t-1)=A^{-1} P_{b}(t \mid t) A^{-1^{\mathrm{T}}}+A^{-1} Q(t) A^{-1^{\mathrm{T}}}
$$

Kalman gain matrix:

$$
K_{b}(t-1)=P_{b}(t-1) C^{\mathrm{T}}\left[C P_{b}(t-1) C^{\mathrm{T}}+R(t-1)\right]^{-1}
$$

State estimation a posteriori:

$$
\hat{x}_{b}(t-1 \mid t-1)=\hat{x}_{b}(t-1)+K_{b}(t-1)\left[y(t-1)-C \hat{x}_{b}(t-1)\right]
$$

Error covariance a posteriori:

$$
P_{b}(t-1 \mid t-1)=P_{b}(t-1)-K_{b}(t-1) C P_{b}(t-1)
$$

Initial conditions:

$$
\hat{x}_{b}(N)=\hat{x}(N \mid N) \quad P_{b}(N)=P(N \mid N)
$$

Applying this algorithm, a new state estimate for $t=N$ through $t=0$ has been computed, and, consequently, the estimate of the friction force from time $t=N$ up to $t=0$ has been obtained using the second equation of (26).

Running forward in time and backward in time the Kalman filter algorithm, we have obtained an estimate of the static property of the disturbance that acts on the system, which was not known; with this additional information, it is possible to estimate the state of the system in a proper way using a standard forward in time Kalman filter for systems with known inputs.

\section{Third step (known input Kalman Filter)}

State estimation a priori:

$$
\hat{x}(t+1)=A \hat{x}(t \mid t)+B F_{f}(t+1)+E(t)
$$

Error covariance a priori:

$$
P(t+1)=A P(t \mid t) A^{\mathrm{T}}+Q(t)
$$

Kalman gain matrix:

$$
K(t+1)=P(t+1) C^{\mathrm{T}}\left[C P(t+1) C^{\mathrm{T}}+R(t+1)\right]^{-1}
$$

State estimation a posteriori:

$$
\hat{x}(t+1 \mid t+1)=\hat{x}(t+1)+K(t+1)[y(t+1)-C \hat{x}(t+1)]
$$

Error covariance a posteriori:

$$
P(t+1 \mid t+1)=P(t+1)-K(t+1) C P(t+1)
$$

Initial conditions:

$$
\hat{x}(0)=\hat{x}_{b}(0) \quad P(0)=P_{b}(0)
$$

Finally the estimation of the friction force can be computed as

$$
\hat{F}_{f}(t)=B^{-1}[\hat{x}(t+1)-A \hat{x}(t)-E(t)] .
$$

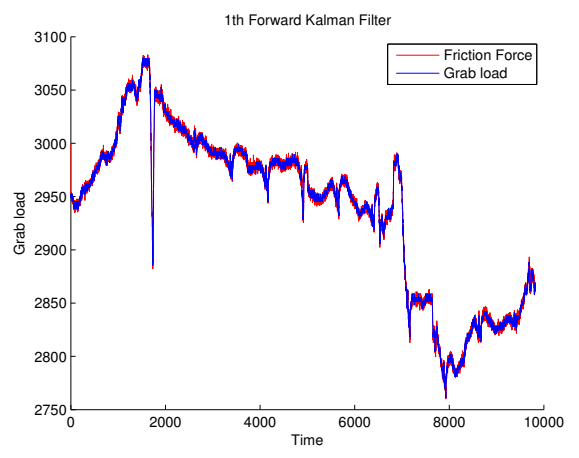

Fig. 1. First iteration estimation.

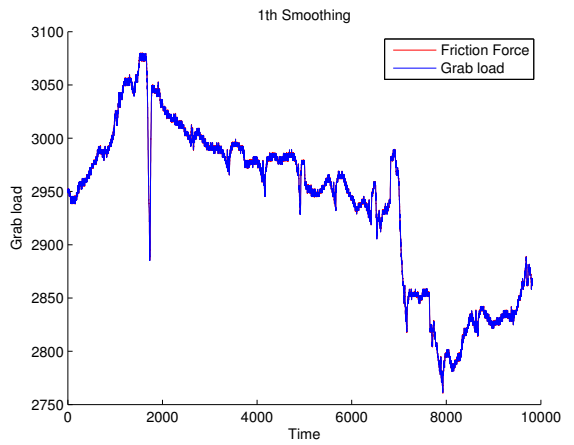

Fig. 2. Second iteration estimation.

\section{SIMULATION RESULTS OF THE PROPOSED ESTIMATION SCHEME}

The three steps algorithm just explained has been applied to real data stored during refueling operations. In figure 1 is depicted the estimation of the friction force after the first step of the algorithm, i.e. after having applied the unknown input Kalman filter. It is possible to observe that the estimated friction force has the same trend of the grab force, but its shape is not exactly the same. This is due to the fact that statistic property of the disturbance $F_{f}$ are not known for $t=0$. In figure 2 is presented the estimation of the friction force after having applied the Kalman smoothing algorithm. Remembering that the smoother algorithm has the role to propagate the estimation of the friction force from time $t=N$, to time $t=0$, a better result can be obtained by processing the system once more by a forward Kalman filter, where now the statistic property of the friction force for $t=0$ are known, because they are given by the combined use of the first forward Kalman filter and the smoother. The results of this third step are shown in figure 3. In this case the trend and the shape of the estimate friction force are exactly the same as the grab load, and this demonstrates that it is possible obtain an optimal estimation of the acting disturbance without an a priori knowledge on it. Still some small errors in the estimate are present; these imperfections are due to the approximate model of the system used to estimate the friction force. For example the model does not consider the noise introduced by the quantization of data, moreover both the mass $m$ of the fuel assembly and the value of the buoyancy force $F_{a}$ are approximated and considered constant. 


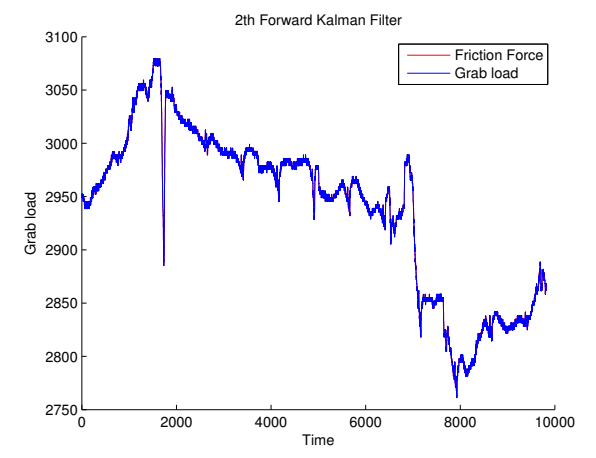

Fig. 3. Third iteration estimation.

\section{DEALING WITH QUANTIZATION}

Aim of this section is to give some guidelines on how to face the problem of state estimation using quantized measurements; this is necessary since grab load data are quantized and the quantization introduce a noise that affect the estimate. In the following some necessary condition for the maximum likelihood estimate (see Raunch et al. [1965]) when the observations have been quantized will be given and a Quantization Regression (QR) algorithm (still based on Kalman filter) which generates an estimate of an autoregressive time series from quantized measurements will be described.

As reported in Widrow et al. [1996], the effect of a uniform quantization can be modeled as an additive noise that is uniformly distributed, uncorelated with the input signal, and has white spectrum. Consider the following model with quantized measurements:

$$
\begin{aligned}
x_{t+1} & =f\left(x_{t}, w_{t}\right) \\
z_{t} & =h\left(x_{t}\right)+e_{t} \\
y_{t} & =Q_{m}\left(z_{t}\right)
\end{aligned}
$$

where $Q_{m}(\cdot)$ is the quantization function. The problem of optimally estimate the state of (41) is a problem of nonlinear non-Gaussian filtering; as explained in Jazwinski [1970] such a problem has a Bayesian solution given by

$$
\begin{aligned}
p\left(x_{t+1} \mid Y_{t}\right) & =\int_{R^{n}} p\left(x_{t+1} \mid x_{t}\right) d x_{t} \\
p\left(x_{t} \mid Y_{t}\right) & =\frac{p\left(y_{t} \mid x_{t}\right) p\left(x_{t} \mid Y_{t-1}\right)}{p\left(y_{t} \mid Y_{t-1}\right)} .
\end{aligned}
$$

In the following a slightly different Kalman filter obtained by the Bayesian equation as shown in Curry [19703] will be introduced; considering this filter, necessary conditions for the maximum-likelihood estimate of parameters when the observations are quantized will be formulated. Consider the following linear measurement equation $z=\mathrm{H} x+v$, where $x$ is the vector to be estimated, $z$ is the measurement vector, and $v$ is the measurement noise. Recall that, with non-quantized measurements, the maximum-likelihood estimate (cf Raunch et al. [1965]) is the value of $x$ that maximizes the likelihood function $L(z ; x)$ :

$$
\hat{x}=\arg \left[\max _{x} L(z, x)\right]=\arg \left[\max _{x} p(z: x)\right],
$$

where the notation $p(z: x)$ means the probability-density function of $z$ with $x$ as a parameter of the distribution.
When the measurements are quantized numerical values of $z$ are not available and the knowledge of the measurements is reflected in the inequalities

$$
\left\{a^{i} \leq z^{i}<b^{i}\right\},
$$

where $a^{i}$ and $b^{i}$ are the lower and upper bounds of the quantum interval in which the $i$-th component of $z$ is known to lie. Considering this fact, the likelihood function to be used is the probability that the measurements fall in the hypercube defined by equation (43):

$$
L\left(a^{i}, b^{i}, x\right)=\prod_{i} P\left[a^{i}-(H x)^{i} \leq v^{i}<b^{i}-(H x)^{i}\right] .
$$

Hence the maximum-likelihood estimate of $x$ with quantized measurements is

$$
\hat{x}=\arg \left\{\max _{x} \prod_{i} P\left[a^{i}-(H x)^{i} \leq v^{i}<b^{i}-(H x)^{i}\right]\right\} .
$$

Denoting as $P_{i}$ the term $P\left[a^{i}-(H x)^{i} \leq v^{i}<b^{i}-(H x)^{i}\right]$, such that $P_{i}=\int_{a^{i}-(\mathrm{Hx})^{i}}^{b^{i}-(H x} p_{v^{i}}(u) d u$, the necessary condition for maximum likelihood estimate is the following:

$$
\begin{aligned}
& \frac{1}{L\left(a^{i}, b^{i}, x\right)}\left(\frac{\partial L\left(a^{i}, b^{i}, x\right)}{\partial x}\right)=\sum_{i} \frac{\partial P_{i} / \partial x}{P_{i}}= \\
& =\sum_{i} \frac{p_{v^{i}}\left(b^{i}-(H x)^{i}\right)-p_{v}^{i}\left(a^{i}-(H x)^{i}\right)}{P_{i}} h^{i}=0,
\end{aligned}
$$

where the row vector $h^{i}$ is the $i$-th row of $H$. Hence the problem can be formulated as following. Given the measurement equation $z=h(x, v)$, the joint probability density function of parameter and noise vectors $p_{x, v}(\xi, v)$, the constraint $z \in A$, where $A$ is some hypercube for quantized measurements, the estimation problem with quantized measurements consists in finding the conditional mean of $f(x)$ given a measurement $z: E[f(x) \mid z]$ and averaging this function of $z$ considering the constraint $z \in A$.

Assume that the state vector and measurements variables satisfy the relationships

$$
\begin{aligned}
x_{i+1} & =\Phi_{i} x_{i}+w_{i} & & \\
z_{i} & =H_{i} x_{i}+v_{i} & & \\
E\left(x_{0}\right) & =\bar{x}_{0} & & \operatorname{cov}\left(x_{0}\right)=P_{0} \\
E\left(w_{i}\right) & =0 & & E\left(w_{i} w_{j}^{T}\right)=Q_{i} \delta_{i j} \\
E\left(v_{i}\right) & =0 & & E\left(v_{i} v_{j}^{T}\right)=R_{i} \delta_{i j} \\
E\left(w_{i} v_{j}^{T}\right) & =0 & & E\left(w_{i} x_{0}^{T}\right)=E\left(v_{i} x_{0}^{T}\right)=0
\end{aligned}
$$

where $x_{i}$ is the system state vector at time $t_{i}, \Phi_{i}$ is the system transition matrix from time $t_{i}$ to $t_{i+1}, w_{i}$ is a realization of the process noise at $t_{i}, z_{i}$ is the measurement vector at time $t_{i}, H_{i}$ is measurements matrix at time $t_{i}$ and $v_{i}$ is a realization of the observation noise at time $t_{i}$. Each of the $m$ components of the normally distributed vector $z$ has zero mean and lies in a interval whose limits are $\left\{a^{i}\right\}$ and $\left\{b^{i}\right\}, a^{i} \leq z^{i}<b^{i},(i=1,2,1 \ldots, m)$. Let $\left(\gamma^{i}\right)$ $(i=1,2,1 \ldots, m)$ be the $m$ components of the geometric center vector $\gamma$ of the region $A, \gamma^{i}=\frac{1}{2}\left(b^{i}+a^{i}\right)$, and let $\left(\alpha^{i}\right)(i=1,2,1 \ldots, m)$ be the $m$ components of the quantum interval half-widths vector $\alpha, \alpha^{i}=\frac{1}{2}\left(b^{i}-a^{i}\right)$. It is possible to show that, expanding the probability-density 


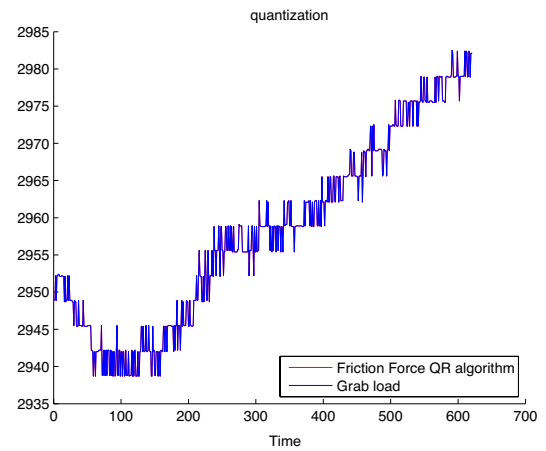

Fig. 4. Estimation of friction force using the modified Kalman filter to deal with quantization (zoom in).

function in power series in an interval containing $\gamma$ and neglecting terms higher than the fourth order, the mean and covariance of $z$ conditioned on $z \in A$ are given by $E(z \mid z \in A) \approx \gamma-A \Gamma^{-1} \gamma$ and $\operatorname{cov}(z \mid z \in A) \approx A=$ $\left\{\frac{\left(\alpha^{i}\right)^{2}}{3} \delta_{i j}\right\}$ where $\Gamma=E\left(z z^{T}\right)$ and $\delta_{i j}$ is the Kronecker delta. In this case the minimum-variance linear estimate $x^{*}$ and its covariance $E^{*}$ are given by

$$
\begin{aligned}
x^{*} & =\bar{x}+K^{*}(\gamma-H \bar{x}) \\
E^{*} & =M-M H^{T}(\Gamma+A)^{-1} H M
\end{aligned}
$$

where $K^{*}=M H^{T}(\Gamma+A)^{-1}$ and $\Gamma=H M H^{T}+R$. This problem can therefore be solved recursively with a modified Kalman filter, leading to the following result. Assuming the conditional distribution of the state just before the $i$-th measurements being $N\left(\hat{x}_{i \mid i+1}, M_{i}\right)$, then the Gaussian fit alghorithm for a linear system with quantized system is the following:

$$
\begin{aligned}
\hat{x}_{i \mid i} & =\hat{x}_{i \mid i-1}+K_{i}\left[E\left(z_{i} \mid z_{i} \in A_{i}\right)-H_{i} \hat{x}_{i \mid i-1}\right] \\
K_{i} & =M_{i} H_{i}^{\mathrm{T}}\left(H_{i} M_{i} H_{i}^{\mathrm{T}}+R_{i}\right)^{-1} \\
P_{i} & =M_{i}-M_{i} H_{i}^{\mathrm{T}}\left(H_{i} M_{i} H_{i}^{\mathrm{T}}+R_{i}\right)^{-1} H_{i} M_{i} \\
E_{i} & =P_{i}+K_{i} \operatorname{cov}\left(z_{i} \mid z_{i} \in A_{i}\right) K_{i}^{\mathrm{T}} \\
\hat{x}_{i+1 \mid i} & =\Phi_{i} \hat{x}_{i \mid i} \\
M_{i+1} & =\Phi_{i} E_{i} \Phi_{i}^{T}+Q_{i}
\end{aligned}
$$

where $\hat{x}_{i \mid i}$ is the conditional mean of $x_{i}$ for quantized measurements up to and including $t_{i}, \hat{x}_{i \mid i-1}$ is the conditional mean of $x_{i}$ for quantized measurements up to and including $t_{i-1}, A_{i}$ is the quantum region in which $z_{i}$ falls, $M_{i}$ is the conditional covariance of $x_{i}$ for quantized measurements up to and including $t_{i-1}, K_{i}$ is the Kalman filter gain matrix at $t_{i}, P_{i}$ is the conditional covariance of estimate, and $E_{i}$ is the conditional covariance of $x_{i}$ for quantized measurements up to and including $t_{i-1}$. The algorithm just proposed has been applied to the AGR monitoring problem and a set of simulations has been performed; the results are presented in figure 4 . It is possible to see that now the estimation algorithm leads to a perfect estimate of the friction force.

\section{CONCLUDING REMARKS}

In this work we have presented a 3-step estimation algorithm based on Kalman filter to monitor the integrity condition of the core of AGR nuclear stations. The following conclusions can be drawn:

- it is possible to estimate the friction force that the fuel rods apply on the supporting brushes;

- as a consequence it is possible to estimate the shape of the graphite bricks that compose the core and therefore the integrity condition of the core itself.

More work needs to be done in order to check the robustness of the approach, gathering existing and historical data in a single location and defining patterns for verifying whether time, location, operating condition have a misleading effect on the trace.

\section{REFERENCES}

C. Bonivento. On line state estimation and indirect measurement in safeguards systems. In: F. Argentesi, $R$. Avenhaus, M. Franklin and J.P. Shipley (eds), Mathematical and statistical methods in nuclear safeguards. Harwood academic publisher, 1983.

C. Bonivento, M.J. Grimble, L. Giovanini, M. Monari, and A. Paoli. Estimation approach to monitoring AGR nuclear reactor. In: G. Picci and M.E. Valcher (eds), A tribute to Antonio Lepschy. Edizioni Libreria Progetto Padova, 2007.

R. Curry. Estimation and control with quantized measurements. The MIT Press, 19703.

H. Emara-Shabaik. Filtering of linear systems with unknown inputs. Journal of dynamic systems, measurments and control, 29:482-485, 2003.

M.S. Grawal and A.P. Andrews. Kalman filtering theory and practice. Prentice Hall, 1993.

A.H. Jazwinski. Stochastic processes and filtering theory. Mathematics in science and engineering, 1970.

Y. Pang, L. Giovanini, and M.J. Grimble. Condition monitoring of a advanced gas-cooled nuclear reactor core. Technical report, British Energy, 2006.

Y. Pang, M.J. Grimble, A.W. Ordys, and J. Reed. Condition monitoring of the nuclear graphite core using benchmarking techniques. In Proceedings of European Control Conference, Kos, Greece, 2007.

H.E. Raunch, F. Tung, and C.T. Striebel. Maximum likelihood estimates of linear dynamical systems. AIAA Journal, 3(8), 1965.

G.M. West, G.J. Jahn, D.J. McArthurand, and J. Reed. Graphite core condition monitoring through intelligent analysis of fuel grab load trace data. Technical report, British Energy, 2005a.

G.M. West, S.D.J. McArthur, J.R. McDonald, A. Ballantyne, J. Reed, and S. Beynon. Graphite core brick crack detection through automated load trace analysis. Technical report, British Energy, 2005b.

B. Widrow, I. Kollar, and M.C. Liu. Statistical theory of quantization. IEEE Transactions on Instrumentation and Measurments, 45:353-361, 1996.

I. Ziskand and D. Hertz. Multiple frequencies and ar parameters estimation from one bit quantized signal with the em algorithm. IEEE Transactions on Signal Processing, 41:3202-3206, 1993. 